\title{
PROSPECCIÓN MAGNETOTELÚRICA DE ACUÍFEROS Y ESPESOR BASÁLTICO EN CARLOS PELLEGRINI, PROVINCIA DE SANTA FE (ARGENTINA)
}

\author{
MANUEL MAMANI, ${ }^{1,}$, BIBIANA CASTIGLIONE ${ }^{2} \&$ ORLANDO DI GIUSEPPE $^{1}$
}

\begin{abstract}
Resumo En el presente trabajo se realizaron tres sondeos magnetotelúricos en la localidad de C. Pellegrini, provincia de Santa Fé, formando un perfil Oeste-Este de 5926 m de extensión. El objetivo fue el estudio del subsuelo, con la finalidad de determinar la posible presencia de acuíferos bajo una cubierta basáltica y el espesor basáltico de esta región del país perteneciente a la denominada Cuenca Chacoparanense. Estas efusiones volcánicas alcanzaron una gran extensión (1,2 millones de km2) y fueron contemporáneas con la apertura continental. Los resultados permitieron identificar cuatro formaciones. La primera de ellas posee baja resistividad, de 2 a $4 \Omega \mathrm{m}$ (excepto los primeros $100 \mathrm{~m}$ con $10 \Omega \mathrm{m}$, indicando la presencia de material sedimentario moderno). Este estrato tiene continuidad hasta una profundidad de $700 \mathrm{~m}$ al Oeste y $850 \mathrm{~m}$ al Este del perfil. La segunda formación se trata, según nuestra interpretación, del estrato basáltico constituido por la Formación Serra Geral, de $680 \mathrm{~m}$ de espesor en el Oeste y 390 $\mathrm{m}$ en el Este y resistividades comprendidas entre $50 \Omega \mathrm{m}$ y $150 \Omega \mathrm{m}$. Por debajo son visibles depósitos sedimentarios con 1-7 $\Omega \mathrm{m}$ de resistividad, donde se pueden discriminar diversos horizontes de material sedimentario, que tiene el tope en $1200 \mathrm{~m}$ de profundidad aproximadamente y su base llega hasta $2400 \mathrm{~m}-3000 \mathrm{~m}$ de profundidad. Dentro de este potente estrato se distingue una capa basal de $30 \Omega \mathrm{m}$ y espesor aproximado de $200 \mathrm{~m}$, que sigue la geometría de lo que interpretamos como basamento cristalino. Este último tiene resistividades mayores a $100 \Omega \mathrm{m}$ y se trataría de una formación masiva que se corresponde con la edad Precámbrica y forma parte del cratón continental.
\end{abstract}

Palabras Claves: Geofísica; Magnetotelúrica; Espesor Basáltico.

\begin{abstract}
MAGNETOTELLURIC INVESTIGATION OF AQUIFERS AND BASALTIC THICKNESS IN CARLOS PELLEGRINI, SANTA FE PROVINCE (ARGENTINA). Three magnetotelluric soundings were performed in C. Pellegrini, Santa Fe Province determining a West-East profile of $5926 \mathrm{~m}$ of long. The aim of the study was the estimation of possible aquifers underneath a basaltic cover. The basaltic thickness in this area of the Argentina corresponding to the Chacoparanense Basin was also estimated. These lava overfloors reached a great extension $(1,2$ million $\mathrm{km} 2)$ and were contemporary with the continental aperture. Results allowed the identification of four stratigraphic formations. The first one has low resistivity of 2 to $4 \Omega \mathrm{m}$ (except the first $100 \mathrm{~m}$ with about $10 \Omega \mathrm{m}$; which corresponds to modern sediments). This formation is present up to $700 \mathrm{~m}$ depth westwards of the profile and 850 $\mathrm{m}$ eastwards. The second formation is a basaltic layer, (Serra Geral Fm), with $680 \mathrm{~m}$ thickness in the West and $390 \mathrm{~m}$ and East of the profile, and resistivities between $50 \Omega \mathrm{m}$ to $150 \Omega \mathrm{m}$. The following formation corresponds to sedimentary rocks showing a resistivity of 1-7 $\Omega \mathrm{m}$, where different sedimentary horizons can be discriminated; its top is approximately at $1200 \mathrm{~m}$ depth and the base reaches the range of depth of $2400 \mathrm{~m}$ to $3000 \mathrm{~m}$. Within this think layer, a basal cover of $30 \Omega \mathrm{m}$ can be distinguished with approximately $200 \mathrm{~m}$ thickness, following the geometry of what can be interpreted as crystalline basement. Finally a last block has resistivities higher than $100 \Omega \mathrm{m}$ and it may be a massive formation corresponding to Precambrian age, as part of the continental craton.
\end{abstract}

Keywords: Geophysics; Magnetolluric; Basaltic Thickness.

INTRODUCCIÓN Con el objetivo de estudiar la presencia de acuíferos profundos que podrían servir como recurso para el aprovechamiento de aguas termales ó como agua potable en otro caso y, en forma concomitante con ello, investigar los estratos suprayacentes determinando el estado, resistividad y espesor de los mismos que tienen composición basáltica y que cubren gran parte del litoral argentino, se realizaron en cercanías de la localidad de C. Pellegrini y sobre la ruta provincial $\mathrm{N}^{\circ} 13$, en la Prov. de Santa Fe, tres sondeos exploratorios magnetotelúrico en una dirección Oeste - Este (ver Figura 1). Los mismos permitieron alcanzar una profundidad de investigación tal que sirvió para identificar al espesor sedimentario de la zona, discriminar la presencia de acuíferos en el área de estudio y estudiar, dentro del craton continental, la existencia de estructuras de baja resistividad. Se logró información que permitió interpretar, mediante el procesamiento adecuado, datos del subsuelo mas profundo de lo conocido hasta el momento, discriminando los estratos de mayor espesor y en especial el que se corresponde con el basalto, existente en la región y que forma parte de la Formación Serra Geral.

Además este estudio nos permitió determinar la geometría y la profundidad del basamento cristalino, atravesando, con el método geofísico utilizado, la pantalla sísmica que significa la presencia de las coladas volcánicas y además lograr información en un área donde, debido a la conductividad presente, no permite una profundización por otros métodos geofísicos (eléctricos en particular).

Estos trabajos se realizaron para el proyecto SEGEMAR (Servicio de Geología y Minería de Argentina) - Municipio de C. Pellegrini que tiene como finalidad, prospectar el subsuelo de la localidad para evaluar la posibilidad de detectar fluidos termales.

Esta gran cuenca sedimentaria no ha tenido en su historia geológica grandes eventos tectónicos; por lo tanto, las manifestaciones superficiales son escasas para lograr una interpretación de la evolución y el estado final. Luego, las informaciones del subsuelo que muestren resultados y que puedan ser correlacionables con los datos existentes, siempre son de utilidad. En ese concepto se inscribe este trabajo, que incorpora al estado del conocimiento, datos del borde occidental de los derrames lávicos, espesor, profundidad y resistividad de los depósitos sedimentarios subyacentes, los cuales son capaces de albergar agua en

1 - IANIGLA CONICET, Centro Regional de Investigaciones Científicas y Tecnológicas, Av. Ruiz Leal s/n Parque General San Martín. Mendoza, Argentina. CP 5500. E-mail: mmamani@lab.cricyt.edu.ar

2 - Facultad de Ingeniería, Universidad Nacional de Cuyo, Centro Universitario, Parque Gral. San Martín - Mendoza CP5500. E-mail: bcasti@uncu.edu.ar 


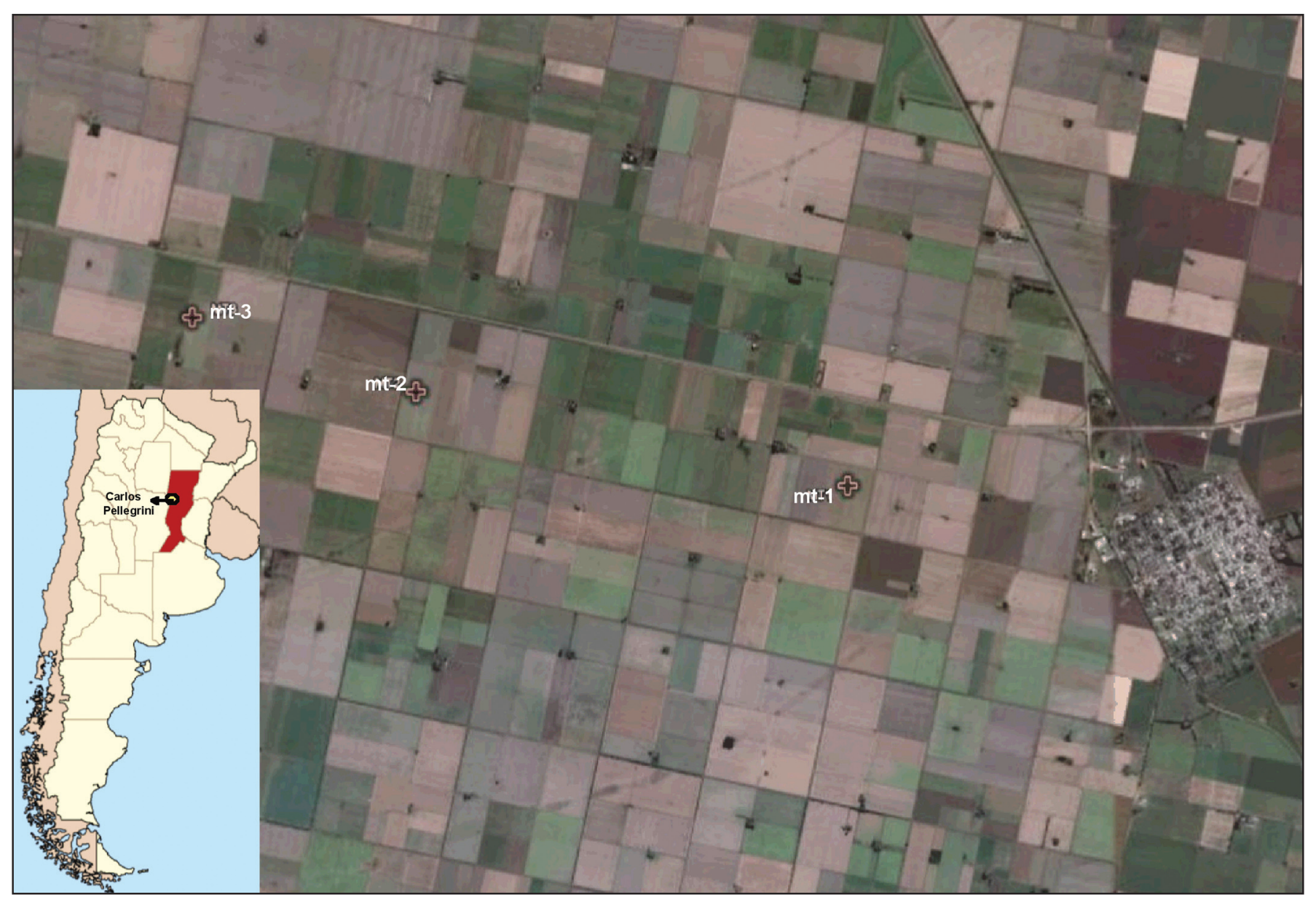

Figura 1: Zona de estudio y ubicación de sondeos ó sitios de mediciones magnetotelúricas

acuíferos con capacidad de ser aprovechados.

ANTECEDENTES Los sitios de medición se encuentran en el lado Oeste del área de mayores desarrollos de los basaltos de edad Mesozoica y pertenecen a lo que se conoce en la literatura como Cuenca Neopaleozoica Chacoparanense (Chebli et al. 1999). Estos basaltos, incluidos en la pila sedimentaria, provienen de las efusiones basálticas toleíticas de la formación Serra Geral y han sido contemporáneas con la sedimentación eólica y fluvial. La Cuenca Chacoparanense que cubre territorios de Brasil, Paraguay, Uruguay y Argentina, está constituida por un gran depósito sedimentario, y se encuentra ubicada en la región Centro-Este de Sud América teniendo su mayor desarrollo durante el Paleozoico y el Mesozoico. Las efusiones basálticas cubren un área de aproximadamente 1.2 millones de $\mathrm{km}^{2}$ y fue formada durante la apertura de océano Atlántico Sur en el Cretácico temprano.

En toda el área cubierta, que abarca territorios de los cuatro países, el flujo de lava es predominantemente basalto toleítico y basalto andesítico en un $90 \%$, así como también bajos niveles de andesitas, lati andesitas, lutitas, riolitas. Un estudio petrográfico y mineralógico realizado en la zona del Sur de Brasil (Schenato et al. 2003), sobre un basalto toleítico, determina que sobre una pila de un estrato basáltico de $45 \mathrm{~m}$, se pueden distinguir tres zonas. Una primaria de bajo contenido vesicular con dimensiones de hasta 5 a $20 \mathrm{~cm}$ de espesor que tiene $5 \%$ de vesículas, que es la parte de la pila que se pone en contacto inmediatamente luego de extruído el basalto con el estrato subyacente, sedimentario en este caso, la segunda zona que es interior, masiva, de $30 \mathrm{~m}$, donde hay ausencia de vesículas y la zona superior (de $15 \mathrm{~m}$ ) que tiene un regular patrón de distribución vesicular, alrededor del $20 \%$ y es la que esta en contacto, cuando es eructada, con el ambiente, y pasa abruptamente de liquido (a $1200^{\circ} \mathrm{C}$ ) a sólido $\left(\right.$ a $980^{\circ} \mathrm{C}$ ) que es cuando se produce la mayor pérdida de gases y es lo que provoca la porosidad manifestada.

El origen del fuerte magmatismo de la región coincide con el rifting que dio origen al apartamiento de América del Sur del África. Las tensiones que se originaron en los cratones y la resistencia a esta apertura, provocaron el agrietamiento en la litosfera y la elevación de la astenosfera, que dio lugar a la salida de volúmenes significativos de rocas volcánicas que cubrieron los estratos sedimentarios del norte de Brasil y de la cuenca Chacoparanense (Thomaz Filho et al. 2000). La respuesta intracratonica a la ruptura, trajo como consecuencia que exista agrietamiento hacia dentro de la cuenca y hasta a considerables distancias del punto de separación, así también hubo zonas con menor extrusión de rocas volcánicas, o donde inclusive no hubo agrietamiento, de esta forma las coladas que llegaron hasta esos sitios mas alejados, lo hicieron con menores volúmenes, luego con menor desarrollo vertical.

La mayor intensidad de la actividad magmática intrusiva y extrusiva ocurrida en el Paleozoico intracratonico se produjo en el periodo Mesozoico y entre ellos, el Triásico, el Jurásico y el Neocomiano y continuó con menor intensidad en el Eoceno y Oligoceno. De modo que la cuenca ha sido controlada por fallas de tipo extensionales y movimientos de subsidencia que dieron lugar a levantamientos marginales e internos (Pezzi \& Mozetic 1989), la fuerte erosión en diversas categorías fue rellenando esos bajos hasta llegar a la geometría actual.

La cuenca sedimentaria del Paraná ha tenido durante la etapa 
del Fanerozoico reactivaciones del basamento Cratónico (Ribeiro et al. 2004). Durante los periodos Paleozoico y Mesozoico los diversos episodios ocurridos dieron lugar a las sucesivas etapas de acumulación de sedimentos, que tenían por su parte sucesivos periodos de erosión y que además fueron afectados por tectónicas particulares y por el clima imperante. La reactivación del basamento, en uno de los episodios descriptos por Ribeiro (2004), dentro de las fases tectónicas, ocurrió en el Cretácico temprano, y que dio lugar al levantamiento de la isoterma (aumento de calor) relacionado con la apertura continental, y es el momento donde ocurre el episodio del magmatismo basáltico Toleítico de la Formación Serra Geral.

En el desarrollo de la cuenca, bajo los niveles basálticos descriptos, quedaron depósitos sedimentarios donde se pueden distinguir tres niveles de acuíferos termales en la región, con diferente desarrollo y presencia. El primero de ellos, correspondientes al Pérmico bajo, es la Formación San Gregorio, equivalente a la Formación Charata en Argentina, que esta compuesta de arenas fina y media y conglomerados. Al fondo de este nivel las rocas sedimentarias fueron depositadas, durante una trasgresión marina (Pesce 2002). El segundo nivel, que es él más importante de toda la serie y que cubre la mayor parte de la superficie se visualiza en las Formaciones Rivera y Tacuarembo, que en territorio de Uruguay constituyen la principal área de recarga del acuífero de nivel medio, explotados en ambos lados del río Uruguay y que se extienden hacia el Oeste, dentro de Argentina. Estos acuíferos son los que dan lugar al denominado acuífero Güaraní y que se encuentra incipientemente explotado, sobretodo para la provisión y el aprovechamiento de las aguas termales.

Sobre antecedentes geofísicos donde la variable a medir sea la resistividad, es de mencionar los valores obtenidos e informados por Menezes \& Travassos (2004) de una perforación realizada en el Arco de Ponta Grossa, donde se determinó la resistividad, dando valores de $130 \Omega \mathrm{m}$ para los basaltos de Serra Geral y valores menores de hasta $100 \Omega \mathrm{m}$ en otros sitios, es decir se encuentra dentro del rango de medidas que se esperan para estos basaltos intruídos con areniscas. Otro antecedente de mediciones geofísicas es realizado, en el sitio de medición magnetotelúrica (MT), con la metodología de los Sondeos Eléctricos Verticales y que en un total de tres sondeos realizó el SEGEMAR, con una apertura de los electrodos de corriente hasta $10 \mathrm{~km}$. Con esta disposición electródica lograron visualizar el estrato basáltico a profundidades cercanas a $750 \mathrm{~m}$ y $850 \mathrm{~m}$ según los sitios.

También existen en la costa Oeste del Río Uruguay mediciones geoeléctricas realizadas por la ex - empresa estatal Agua y Energía (1987) y por las diversas municipalidades del área que tuvieron como objetivo la búsqueda de aguas termales para su aprovechamiento como balneoterapia en particular.

DESARROLLO Durante el mes de enero del año 2005, se realizaron los sondeos magnetotelúricos sobre la ruta 13 y hacia el costado Oeste del ingreso al Municipio de C. Pellegrini (Provincia de Santa $\mathrm{Fe}$ ), abarcando una distancia entre el primer y último sondeo de $5926 \mathrm{~m}$. La cantidad de sitios de exploración fue de tres sondeos y con una orientación que permitió realizar el procesamiento y la interpretación de tal modo de conformar un perfil O-E ( $\left.\mathrm{Rb}: 100^{\circ}\right)$, paralelo a la ruta 13 con las siguientes coordenadas:

La disposición del instrumental y los sensores sobre el terreno, fueron ubicados en sitios donde los ruidos culturales debían ser mínimos, por lo que debieron ser usados terrenos alejados de la ruta 13. Se registraron cinco series temporales en cuatro bandas de frecuencias, solapadas entre sí, formando un rango de frecuencia que va desde $0.0006-250 \mathrm{~Hz}$ lo que aseguraba recabar información del terreno que debería atravesar todo el
Tabla 1: coordenadas de los sitios de medición

\begin{tabular}{|c|c|c|c|}
\hline Sondeo & Latitud & Longitud & H [m] \\
\hline $\mathrm{mt}-1$ & $\mathrm{~S} 32^{\circ} 02^{\prime}, 64$ & $\mathrm{~W} 61^{\circ} 49^{\prime}, 46$ & 106 \\
\hline $\mathrm{mt}-2$ & $\mathrm{~S} 32^{\circ} 02^{\prime}, 15$ & $\mathrm{~W} 61^{\circ} 52^{\prime}, 13$ & 115 \\
\hline $\mathrm{mt}-3$ & $\mathrm{~S} 32^{\circ} 01^{\prime}, 75$ & $\mathrm{~W} 61^{\circ} 53^{\prime}, 54$ & 106 \\
\hline
\end{tabular}

paquete sedimentario del subsuelo.

METODOLOGÍA El método geofísico de exploración empleado fue el denominado Magnetotelúrico (Berdichevsky \& Dmitriev, 1976). Es una metodología de exploración que usa el campo electromagnético natural y considera un frente de onda plana como condición necesaria (Vozoff 1991). La fuente de excitación la constituyen las variaciones del campo geomagnético y las corrientes que asociadas con ellas que circulan a través de la corteza terrestre. No tiene límites en la profundidad de investigación, solo a través de un cambio de sensores magnéticos asociado al distinto tiempo de muestreo, se puede obtener información de cualquier profundidad del subsuelo. Los períodos de las fluctuaciones se extienden desde milisegundos hasta varios años.

La forma de trabajo consiste en medir sobre el terreno la variación de las componentes horizontales del campo magnético y su correspondiente campo eléctrico asociado. Es una metodología pasiva de investigación, en la cual se registran simultáneamente, sobre la superficie de la tierra, las variaciones de las tres componentes del campo geomagnético $(\mathrm{Hx}, \mathrm{Hy}, \mathrm{Hz})$. Las direcciones de medición $x$ e $y$ corresponden a las direcciones N-S y E-O respectivamente, siendo la dirección $z$ la correspondiente a la componente vertical del campo geomagnético. También se registran las variaciones de las componentes del campo eléctrico asociado (Ex,Ey).

A través del procesamiento y el análisis de los datos almacenados en el terreno, se calcula el tensor de impedancias (Bailey \& Groom 1987) así como también la función transferencia para cada periodo. El equipamiento empleado fue el EMI que consta de tres barras de inducción magnética para registrar las componentes del campo magnético, a saber E-W, N-S y el campo vertical, y también cuenta con dos pares de electrodos impolarizables de $\mathrm{Cu}-\mathrm{CuSO}_{4}$ que miden los componentes eléctricos en las dos direcciones, dispuestos en el campo de tal manera de formar dos dipolos eléctricos $(x$ e $y$ ), con los acondicionadores de señales para cada campo en particular.

La debilidad de las señales naturales del orden de $1 \gamma\left(10^{-}\right.$ ${ }^{9}$ teslas) para el campo magnético y $4 \mathrm{mv} / \mathrm{km}$, para el campo eléctrico, condicionan la complejidad del equipo de registro y obliga a una amplificación de las señales que es sensible a los ruidos "culturales". En resumen, para este caso se registraron cinco series temporales, correspondientes a las variaciones de las componentes ortogonales magnéticas y eléctricas en un rango de frecuencias que va desde los $250 \mathrm{~Hz}$ a $0,0006 \mathrm{~Hz}$

De los registros obtenidos y almacenados en un sistema apropiado, se puede calcular a través de un procesamiento, la resistividad aparente y las fases. Como resultado se obtiene un perfil geoeléctrico, que además de indicar el rumbo de las estructuras del subsuelo, puede también indicar la profundidad donde se producen los contrastes de resistividad. A través de un análisis espectral robusto, de las cinco series temporales registradas, se estiman las componentes del tensor de impedancia $Z$ en función de la frecuencia, para cada sondeo. Con los resultados de la impedancia se calculan tanto dirección de rumbo de estructuras principales, como diferentes parámetros para el análisis y corrección de distorsiones galvánicas e inferencias estructurales 1D, 2D, y 3D (Jones 1988). 
En magnetotelúrica, la respuesta de las estructuras regionales puede estar enmascarada por distorsiones producidas por inhomogeneidades locales y/o cercanas a la superficie. Las distorsiones se definen como la alteración de la respuesta regional inductiva debido a la presencia de distribuciones de corrientes y cargas, las cuales producen distorsiones inductivas y galvánicas respectivamente. El efecto inductivo desaparece rápidamente con el período, mientras que no pasa lo mismo con el efecto de las distorsiones galvánicas. Todas las distorsiones afectan a las curvas de resistividad aparente, siendo la fase más estable ante estas distorsiones, sólo en un caso en particular la fase es afectada de una manera temporal y es necesaria su corrección.

Los resultados se pueden visualizar en dos pares de curvas de resistividad aparente y fase, en función de la frecuencia para cada sondeo, tanto en la dirección de medición, como en las direcciones principales de las estructuras, permitiendo obtener a través de un modelado, las resistividades en función de la profundidad, determinando los diferentes estratos subyacentes, bajo cada sitio de medición.

PROCESAMIENTO E INVERSIÓN Luego de almacenar y preparar los registros de terreno para darles el formato adecuado se llevó a cabo el procesamiento de datos, el cual se realizó por un análisis espectral de las series temporales registradas, dando como resultado dos curvas de resistividad aparente y dos de fase provenientes de la ubicación de las dos orientaciones de los sensores en función de la frecuencia, para cada uno de los sondeos magnetotelúricos realizados. Estos dos pares de curvas se repiten tanto en la dirección de medición (N-S y E-O) como a las direcciones de las estructuras principales observadas a través de los resultados del primer procesamiento (Figura 2). Se pueden observar en esta figura los valores de resistividad y fase, con sus respectivas barras de error, llamando TE (transverso eléctrico) cuando las corrientes eléctricas son paralelas a la dirección de rumbo de la estructura y TM (transverso magnético) cuando las corrientes eléctricas circulan en dirección perpendicular al rumbo de la estructura

Con estos resultados y sus ángulos de rotación, que también es un producto del procesamiento, se llevó a cabo la Inversión 2D (dos dimensiones) del perfil, a través de un Programa de Inversión de Relajación Rápida (Smith 1988; Smith \& Booker 1991). Esta forma de Inversión permite observar, entre otras cosas, la influencia que tienen en los resultados obtenidos del procesamiento, las variaciones laterales de la resistividad del terreno que se encuentra entre los sitios de exploración, de modo que el modelo encontrado tiene menores incertidumbres en la traza del perfil, más aun si la estructura se acerca a una estratificación horizontal y homogénea.

Teniendo en cuenta el antecedente geoeléctrico de la zona, obtenidos en el trabajo previo de exploración por el Departamento de Geotermia del Servicio de Geología y Minería de Argentina (SEGEMAR), se aprecia una estratificación horizontal hasta los niveles alcanzados en el subsuelo por los sondeos eléctricos verticales (SEV) definidos también en ellos. Esta situación favorece a una inversión en 2D, pero también en esos resultados se puede observar la gran conductividad de los estratos $(2 \Omega \mathrm{m}$ aproximadamente) yaciendo sobre lo que se presume debería ser la capa basáltica. En estas condiciones, la inversión 2D, a través de este primer programa utilizado, fue de gran dificultad lograr un ajuste en resistividad y en fase (lo deseable) y por ende un modelo óptimo de capas a los efectos de su interpretación. Sin embargo, con esta inversión lo que sí se logró apreciar fue la existencia de un estrato resistivo entre dos conductoras (paquete basáltico), que podía correlacionarse con las curvas de fase, pero no en las que se corresponden con la resistividad. Esta situación se presenta teniendo en cuenta que: a) En la metodología magnetotelúrica, el parámetro más estable ante distorsiones galvánicas o inductivas es la fase y no la resistividad.

b) Para poder inferir esta capa resistiva entre dos estratos conductores es necesario que la misma tenga una potencia de igual o de mayor valor que la suma de los espesores de todos los estratos por arriba de ella (limitación de la metodología).

A partir de ello se estimó que la potencia de este paquete resistivo debía tener un espesor como máximo de $800 \mathrm{~m}$. De este modo, para lograr una inversión 2D apropiada, se utilizó otro programa de inversión que tiene en cuenta las Estructuras Mínimas (Rodi \& Minster 1983).

En primer lugar, se introdujo un modelo inicial que no contemplaba este paquete resistivo. De esta manera, se podría invertir en resistividad correctamente y se confirmó que el modelo respondía a las curvas de resistividad, pero no así a la de fase. A partir de ese momento, es que se tomó la decisión de invertir en fase, por lo anteriormente expresado en el ítems "b", teniendo en cuenta que las curvas de ajuste del modelo de inversión estuvieran, condición necesaria, dentro de los límites del error de los registros. Para ello, se confeccionaron varios modelos iniciales con diferentes espesores para el paquete basáltico (a fin de agilizar la inversión). Luego de varios intentos de inversión iterativa e interactiva se llegó a un modelo final (Figura 3) en el cual se puede observar entre otras cosas el techo o tope del basamento resistivo y la inferencia del estrato que se corresponde con el paquete basáltico. En la Figura 4, se ha clasificado en bloques de importancia y fácilmente observables que permite una aglutinación de lentes, utilizando los valores de la conductividad.

INTERPRETACIÓN En la Figura 3, se observa el modelo resultante de la inversión 2D. En él se pueden diferenciar cuatro paquetes estratificados bien definidos. El primero de ellos, contando desde la superficie, se corresponde con un espesor sedimentario de 2 a $4 \Omega \mathrm{m}$ que tiene continuidad hasta una profundidad de $700 \mathrm{~m}$ al Oeste y $850 \mathrm{~m}$ al Este del perfil. Dentro de este estrato, se diferencia una pequeña capa superficial de unos $10 \Omega \mathrm{m}$ de resistividad de hasta $100 \mathrm{~m}$ de espesor. Esta capa es interpretada como material de suelo seco, según la estratigrafía conocida, limo arcilloso, arenas de distinta granulometría y compactación, donde se encuentra alojado el estrato acuífero de la región (subálveo). Este estrato de mayor resistividad constituiría los sedimentos de edad Cuartaria, seguido luego por los que se corresponden con los de edad Terciaria.

Subyacente al estrato sedimentario mencionado y como segundo elemento distintivo se observa una capa resistiva notable que inferimos se correspondería, por los valores obtenidos, con el paquete basáltico buscado, la llamada Formación Serra Geral, con valores de resistividad $>50 \Omega \mathrm{m}$, hasta cercano a $100 \Omega \mathrm{m}$ en algunos sectores del mismo (bajo mt-2 y mt-3). Esta baja resistividad sobre los valores esperados de un basalto masivo, estimamos se debe a las fracturas secundarias del mismo provocadas en la etapa de enfriamiento e intercalaciones horizontales de material sedimentario. Pero también se debe a la condición física del basalto una vez extruido, teniendo en cuenta a Schenato et al. (2003) para quien el mayor porcentaje en vesículas $(20 \%)$ se encuentra en la parte superior en contacto con el medio ambiente debido al enfriamiento repentino de este material, y del mismo modo, en el sustrato puede tener valores de hasta el $5 \%$ de vesículas. En estas condiciones, en la parte superior se podría haber alojado posteriormente agua debido a la presión hidrostática reinante que logra penetrar en el estrato. Esta situación provoca una menor resistividad del terreno. Esto también se confirma con las mediciones que realiza Menezes \& Travas- 


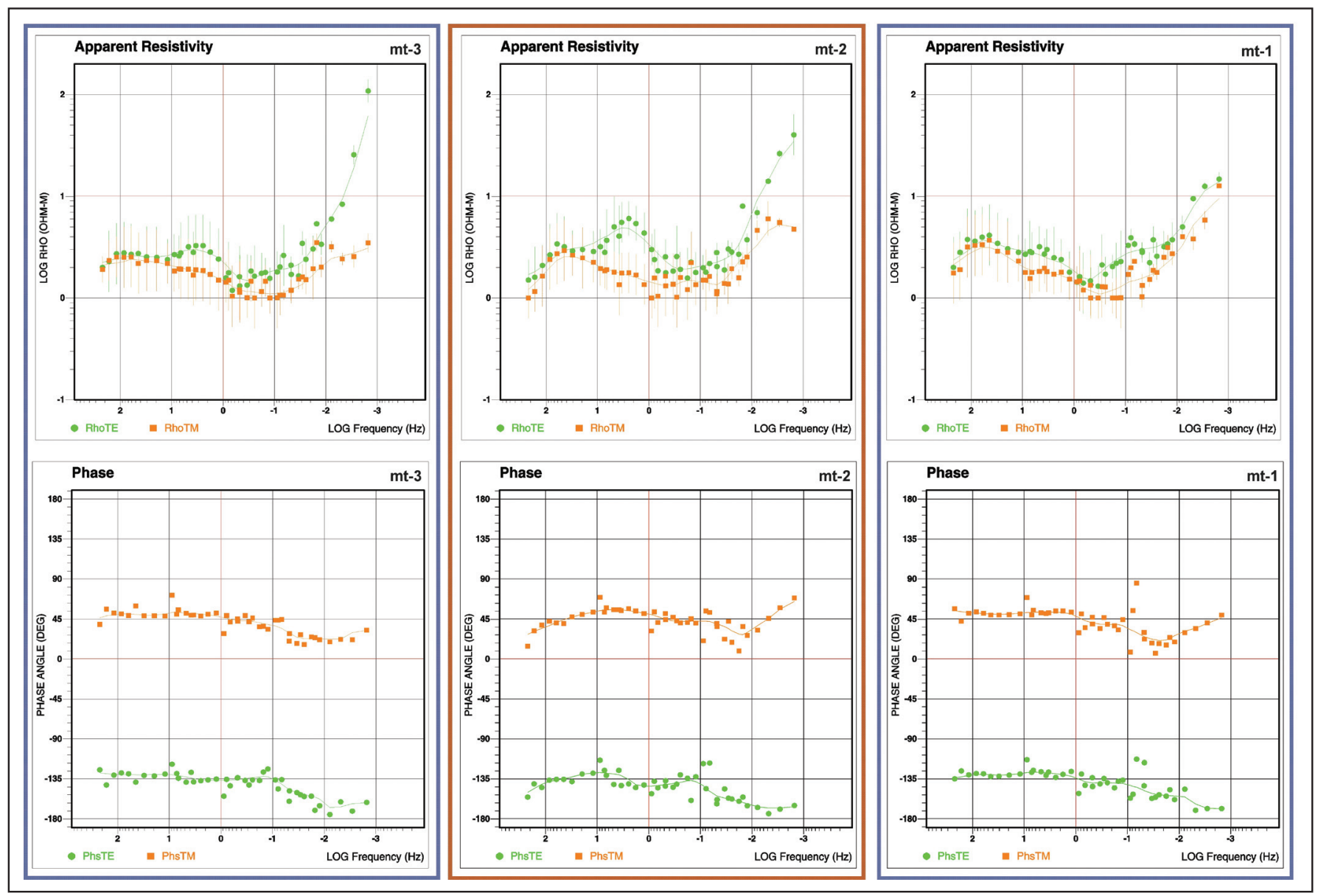

Figura 2: Curvas de resistividad aparente y fase en función de la frecuencia, para los tres sondeos magnetotelúricos realizados.

sos (2004) en el norte de Brasil, que a través de la misma técnica operativa (MT) logra una resistividad similar para el basalto.

Según este modelo presentado, la profundidad del tope del paquete basáltico se encontraría a $850 \mathrm{~m}$ al Este del perfil y a 700 $\mathrm{m}$, aproximadamente al Oeste. Este estrato referenciado como basalto presenta un mayor espesor $(680 \mathrm{~m})$ que incluye todo el estrato diferenciado por la mayor resistividad, bajo el sondeo $\mathrm{mt}-2$, y el menor espesor bajo el sondeo mt-1 (390 m). No es posible discriminar a lo largo del horizonte la presencia de un núcleo masivo en el interior de este estrato por el poco contraste en resistividad, que serviría de cobertor a los posibles acuíferos subyacentes a él, con excepción de la zona entre los sondeos mt3 y $\mathrm{mt} 2$ donde hay sectores que tienen hasta $150 \Omega \mathrm{m}$, seguramente debido a un mayor estado de pureza y masivo del basalto. Sin embargo, esta posible situación, de cobertor, no es dable de producirse por el comportamiento posterior de la colada basáltica en lo que respecta a las fracturas secundarias, de modo que los estratos sellantes deben buscarse por encima del basalto.

Siguiendo la descripción de la Figura 3 y hacia una mayor profundidad, se encuentra el tercer bloque constituyendo un potente estrato de resistividades no muy variables a lo largo de todo el perfil, acotado entre 1-7 $\Omega \mathrm{m}$. En este se incluyen diversos horizontes de material sedimentario, mostrados en la Figura 5 , que tiene el tope en $1200 \mathrm{~m}$ aproximados y su base llega hasta 2400 m y $3000 \mathrm{~m}$ en el otro extremo. Según informe de SEGEMAR se trataría de "...sedimentos pelíticos posiblemente rico en yeso y calizas...". Sin embargo, creemos que es posible discriminar el interior de este bloque, sobretodo teniendo en cuenta los tiempos totales de desarrollo transcurridos desde el Carbonífero hasta el Jurásico, que dieron lugar a los tres niveles descriptos por Pesce (2002) y que se componen de formaciones bien determinadas que fueron depositados en ambientes diferentes y con distinta composición granulométrica. Para el caso de los acuíferos que componen el "nivel medio" de edad Triásico medio a Jurásico temprano, estas formaciones son de tipo fluvial, donde la porosidad llega hasta un 30\% que es lo que permite la mayor acumulación de agua. Además de ello, se debe tener cuenta los distintos grados de cementación que presentan y que tienen que ver con los componentes presentes en el horizonte.

Este bloque presenta la geometría de la base en una forma suavemente ondulada con cambios de poca pendiente y los mayores espesores hacia el Oeste del perfil. Dentro de este potente estrato, se infiere una capa basal de $30 \Omega \mathrm{m}$ (Figuras 4 y 5) de aproximadamente $200 \mathrm{~m}$ de espesor, que tiene continuidad en todo el perfil. Podríamos asumir en concordancia con otros sitios de la región, que se trata de sedimentos paleozoicos asentados, los cuales Pesce (2002) describe como primer nivel dentro del Pérmico bajo a la Formación San Gregorio con correlato de las formaciones Sachajoy y Charata en Argentina, Formación Ordoñez según la información del Pozo Josefina ES -1 (Y.P.F. 1977). Esta capa basal sigue la geometría del último bloque enunciado, que constituiría el basamento final. El espesor encontrado para las formaciones mencionadas, en una perforación realizada en la parte sudeste de la cuenca fue de $292 \mathrm{~m}$ (Almiron) y 95 m en el Sur. También se observan dentro de este bloque distintos sectores más conductivos, así como otros de resistividades mayores de variada forma que seguramente deben cubrir los tres horizontes sedimentarios mencionados. Así se destaca desde bajo el sondeo mt-3 y hacia el sondeo mt-2 e inmediatamente después del paquete basáltico una intercalaci- 


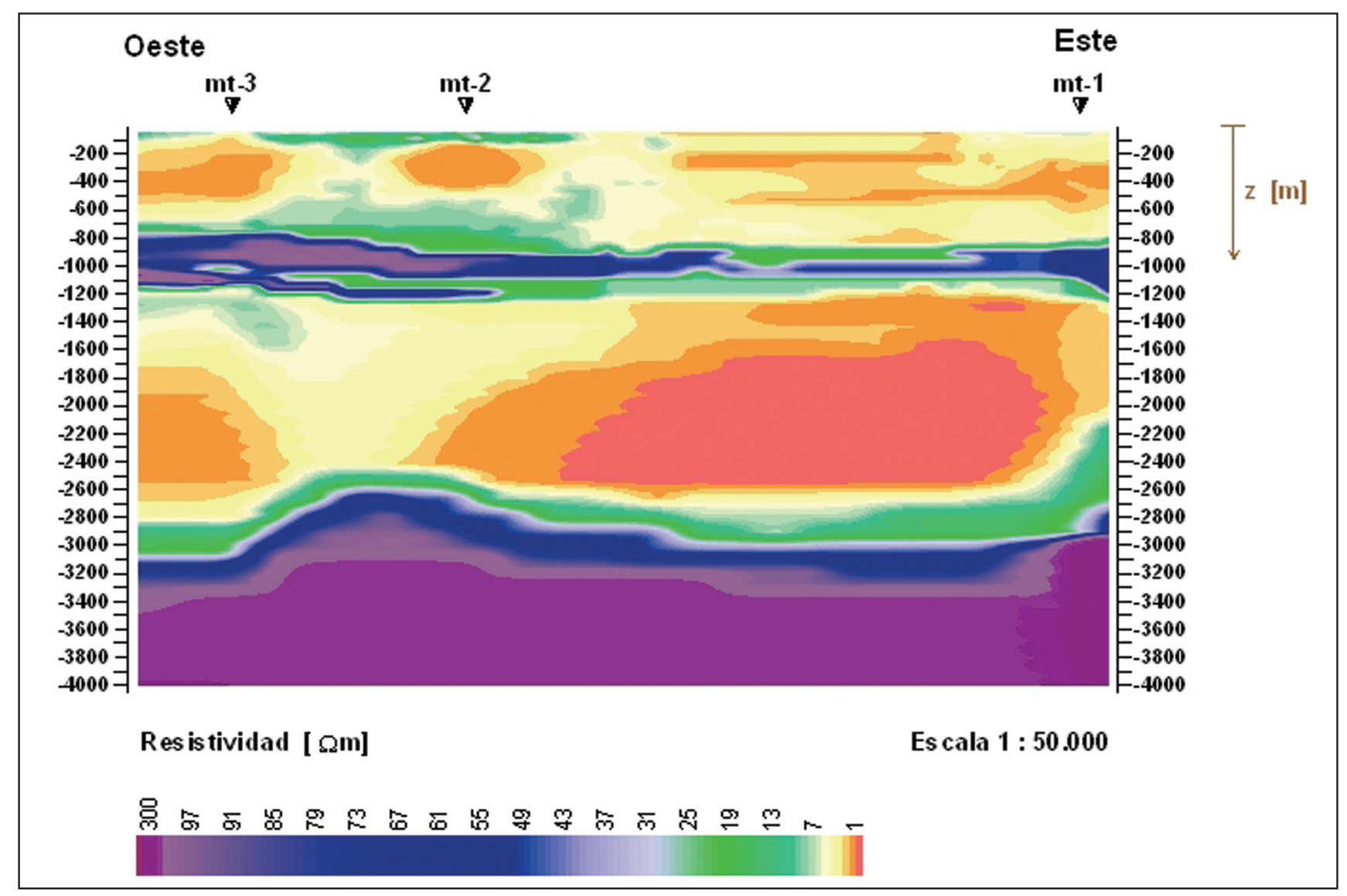

Figura 3: Resultado final de la Inversión magnetotelúrica 2D, del perfil donde se encuentran ubicados los sondeos.

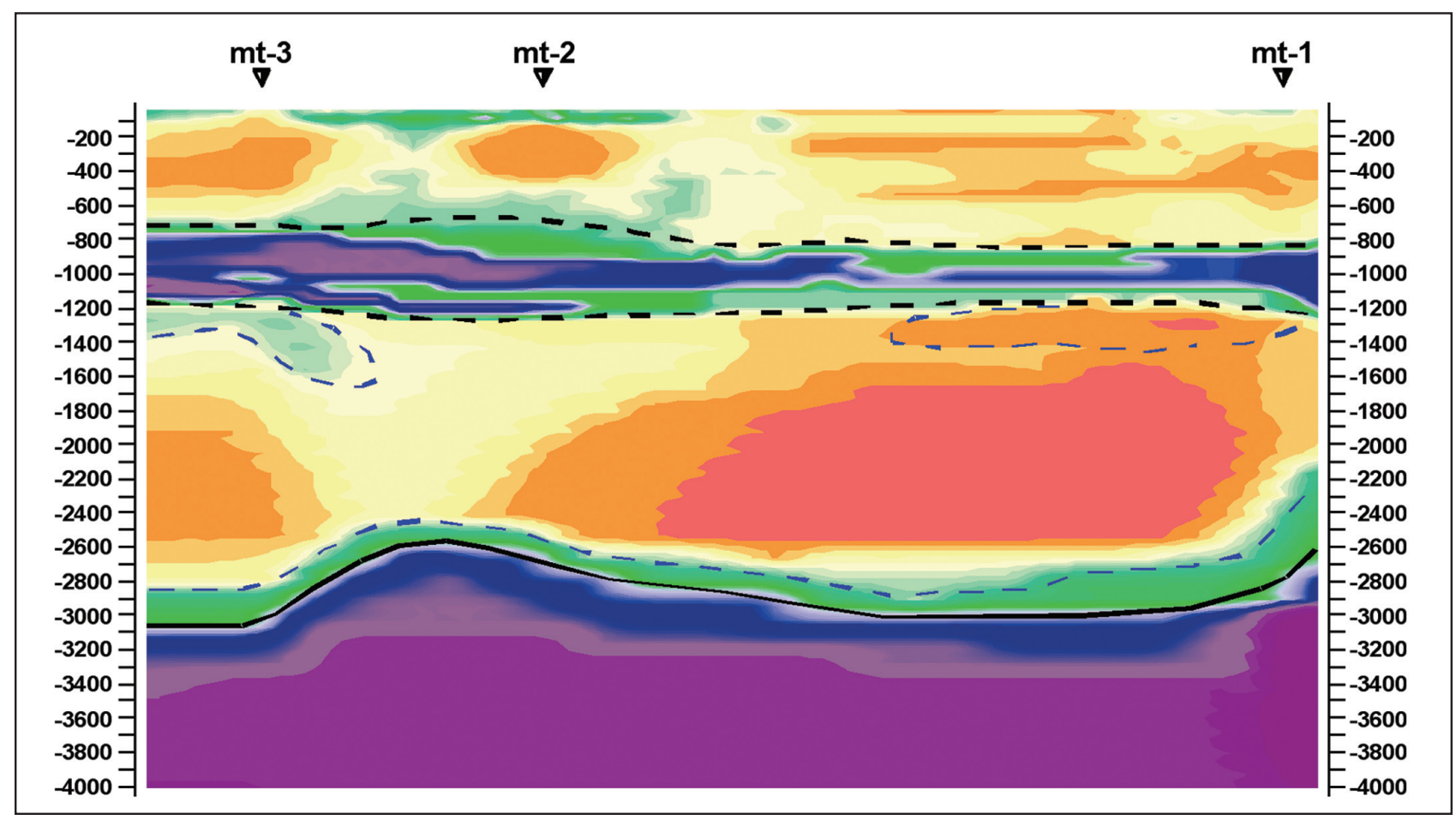

Figura 4: Interpretación de los diferentes estratos resistivos, sobre la inversión 2D realizada presentada en la figura 3.

ón de una pequeña estructura que debe tener su inicio desde el Oeste del perfil y tiene su culminación en esta zona de $10 \Omega \mathrm{m}$ de resistividad; y por el otro extremo, bajo el sondeo mt-1 hacia el sondeo mt-2, se observa una intercalación de conductividad muy alta. Estimamos que la diferencia estriba en el contenido de agua con mayor y menor salinidad además de la conformación 


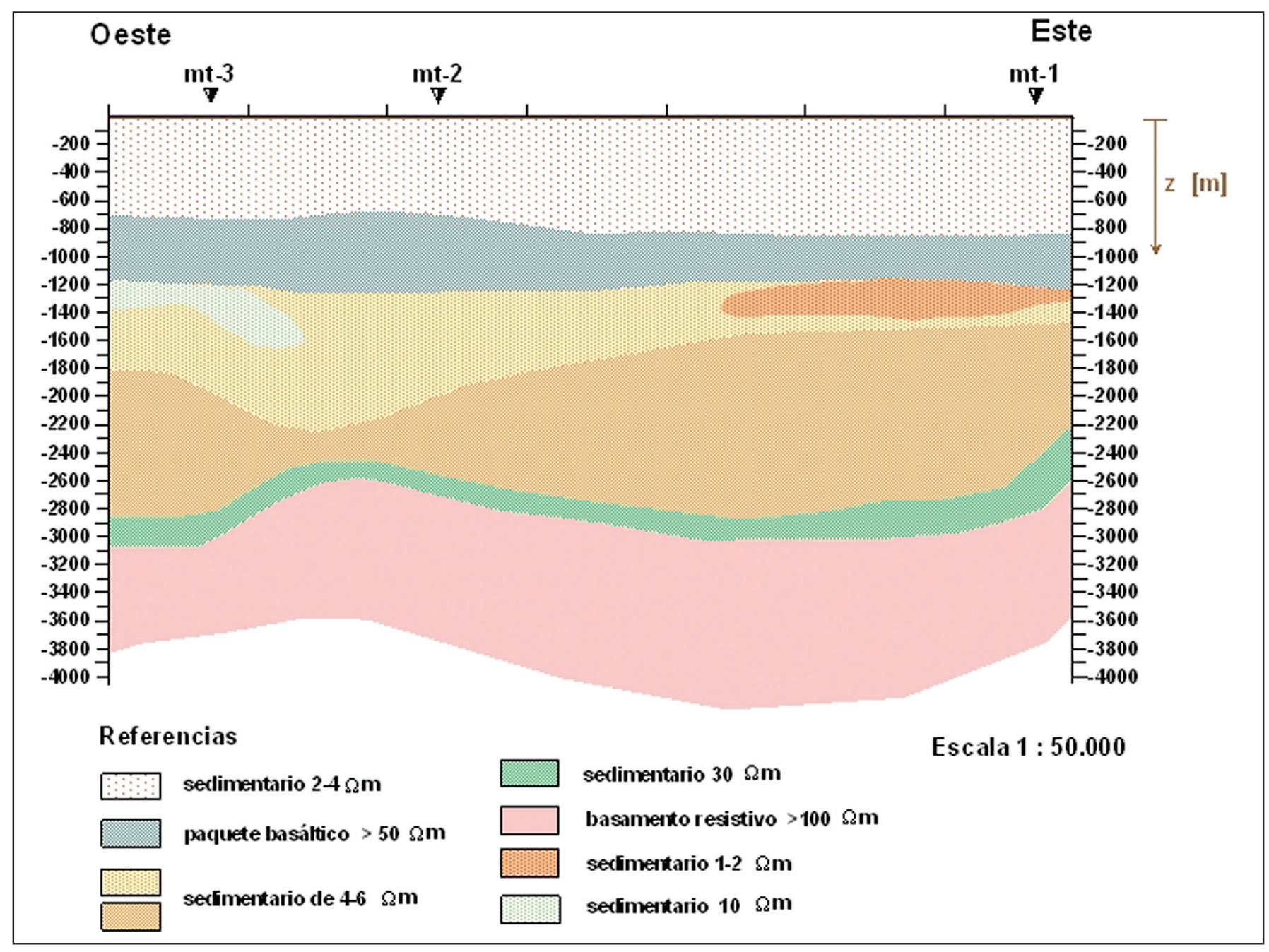

Figura 5: Interpretación final, resultante del estudio magnetotelúrico realizado en la zona.

de los constituyentes del estrato portador del agua. Este estrato se podría asignar a la Formación Tacuarembo, que es la portadora de agua mayormente explotada y hasta donde la mayoría de las perforaciones realizadas en la región pudieron llegar.

También es necesario señalar que la Formación Serra Geral ha sufrido, en su desarrollo, una fracturación intensa, que dio lugar a un fuerte diaclasamiento. Además de ello, se debe considerar los distintos niveles que provienen de las diversas coladas, que dio lugar a los depósitos de material sedimentario intercalado. Esto es observable desde la geometría de lo que nosotros interpretamos como estrato basáltico. Esta situación puede llegar a permitir una interconexión entre los acuíferos por encima y subyacentes de esta Formación, lo cual conformaría acuíferos con mezcla de aguas de diversas líneas de recarga.

El estrato final inferido sería el correspondiente al basamento resistivo, el cual puede observarse en la Figura 4, y mayormente resuelto en su forma en la Figura 5. Este corresponde a resistividades mayores a $100 \Omega \mathrm{m}$ y se trataría de un estrato masivo que tiene la edad Precámbrica y forma parte del cratón continental. Este último bloque descripto se trata del basamento cristalino. De los datos recolectados y utilizando las frecuencias mas bajas (200 s a $250 \mathrm{~s}$ ), se ha logrado invertirlos de modo de lograr una profundidad de investigación de $40 \mathrm{~km}$. Se observa que a esa profundidad prosigue la resistividad obtenida. Y esto marca una diferencia en lo obtenido por Menezes \& Travassos (2004) que en la parte central de Brasil, bajo la cuenca reporta una capa conductora de $30 \Omega \mathrm{m}$ entre 25 y $40 \mathrm{~km}$ en la corteza inferior, que lo atribuye a material hidratado derivado de la acreción en la base de la corteza.

Otro análisis que es posible realizar trata del levantamiento que se observa en el basamento cristalino entre los sondeos $\mathrm{mt} 3$ y $\mathrm{mt}$ 2. En términos de interpretación, seguramente es atribuible a lo que ocurrió en los periodos de reactivación del cratón que dio lugar en otros sitios, al aumento del flujo de calor que provocó el levantamiento de la isoterma del sitio y la elevación del basamento (Ribeiro et al. 2004). En general los valores determinados del gradiente geotérmico son coincidentes con lo esperado en un área continental y han sido calculados entre 0.0208 y $0.024^{\circ} \mathrm{C} / \mathrm{m}$ (Silva Busso 1999).

Por último, en la descripción de las Figuras 4 y 5, en el mismo estrato analizado anteriormente, aparece un sector altamente conductivo entre los sondeos mt-2 y mt-1. Es muy probable que este sector no sea tan conductor como lo indica la inversión, pero si tenga la conductividad del sector que aparece bajo el sondeo mt-3 de $4 \Omega \mathrm{m}$. Esto se debería a la que la separación entre los sondeos mt-2 y mt-1 de $4450 \mathrm{~m}$, supera a la necesaria para una buena inferencia entre ambos sondeos.

CONCLUSIONES Los valores obtenidos del procesamiento y de la interpretación MT tienen correlaciones con lo obtenido en los sondeos geoeléctricos del SEGEMAR, en lo referente a los espesores y a las resistividades obtenidas en el primer bloque sedimentario (2 a $4 \Omega \mathrm{m}$ ). Estos últimos valores en general son muy bajos, lo que impidió seguramente una mayor pene- 
tración de corriente emitida en los sondeos geoeléctricos y por ende definir mejor las estructuras subyacentes de este primer bloque, también dificultó esta situación a la metodología magnetotelúrica durante el procesamiento y el seguimiento de estratos en resistividad. Sin embargo, fue posible discriminar lo que se corresponde con las vulcanitas de Serra Geral, después de un intenso trabajo de procesamiento y la utilización de distintos programas con el objeto de que permita una interacción y una iteración de modo de ajustar a la respuesta "en fase" de los datos magnetotelúricos extraídos del terreno, y al seguimiento de la estratificación por este modo. Eso se logró y permitió la construcción de los modelos presentados. Por otro lado también los valores obtenidos en general son coincidentes con los informados por Morrison (1990).

Es posible correlacionar los valores obtenidos en resistividad, para lo que interpretamos como basaltos tratándose de la misma formación, con los valores obtenidos con el método geoeléctrico (Díaz et al. 1993) en el litoral del Río Uruguay en la Prov. de Entre Ríos. Los resultados para esta Formación están en el orden de $53 \Omega \mathrm{m}, 55 \Omega \mathrm{m}$ y $65 \Omega \mathrm{m}$, según los distintos sitios de medición.

Conocido el perfil estratigráfico del Pozo YPF.SF.Josefina. ES-1, podríamos correlacionarlo con lo obtenido en el perfil geoeléctrico de los sondeos MT. En este caso la zona de mayor interés buscada debería coincidir con lo que se corresponde a los estratos de edad Triásico / Cretácico, de esta forma la parte de mayor resistividad en nuestro segundo bloque analizado se correspondería con la Formación Serra Geral, subyacente a la Formación Mariano Boedo que contiene bancos de yeso y que se visualiza en los diferentes valores de resistividad obtenidos.

De esta forma se logro interpretar el estrato basáltico y, en un modelo $2 \mathrm{D}$, se puede observar la geometría de la base y el tope del mismo, estando compuesto de diferentes espesores y constituyentes, destacándose que se trata de sectores donde esta fracturado e intercalado con lentes de material sedimentario, lo que le confiere una resistividad menor de lo que se espera en un basalto masivo y compacto. También por debajo del mismo se encuentra un potente bloque conductor que seguramente se trata de estructuras de origen sedimentario con resistividades crecientes. Se destaca que, bajo el sondeo mt-1, un cuerpo de hasta $200 \mathrm{~m}$ de espesor que seguramente contiene agua salinizada constituyendo el acuífero que podría ser explotado en una eventual perforación. Asimismo, bajo el sitio donde se realizo el mt-3, se observa otro cuerpo de $10 \Omega \mathrm{m}$ que si se tratara de un acuífero seguramente tiene otras condiciones físico químicas diferentes al anterior que tiene su finalización bajo este sondeo.

Menor incertidumbre (propiedad permanente de todos los métodos de exploración indirectos) se hubiera logrado si se hubiera podido modelar en resistividad y en fase como en un terreno de una estratificación creciente en resistividad y en profundidad y en la forma de 2D. Sin embargo y debido a que la metodología permite hacer este tipo de salvedad, los resultados logrados en el modo fase cubren lo esperado, toda vez que existía y existe un impedimento para aplicar los métodos normales geofísicos eléctricos para la búsqueda de acuíferos que es la baja resistividad. En estas condiciones los resultados deben ser analizados e interpretados con esa salvedad. Los cuerpos sedimentarios de la Figura 5, inmediatos al considerado basalto, tienen una escasa diferencia de resistividad entre ellos (1-2 $\Omega$; 4 4-6 $\Omega \mathrm{m} ;$ y $10 \Omega \mathrm{m}$ ) y también poco desarrollo dentro del espesor total del estrato podemos anticipar que se encuentran en el limite de resolución posible para cualquier método de exploración. Sin embargo y vistos el interés y la significación de este trabajo, nos permitimos interpretar de que se trataría de cuerpos en forma de lentes, con distintos canales de alimentación. Pero, asimismo dentro mismo del estrato basáltico hay intercalaciones de estratos sedimentarios que podrían contener agua, toda vez que la resistividad es menor de lo esperado si se tratara de un estrato masivo, que no es posible diferenciar dado el poco espesor para esta metodología.

Otro elemento de valor es la definición de lo que se llama el basamento cristalino, quizás no para este evento que tiene su propio interés termal, pero si como conocimiento geológico debido a que se trataba de un interrogante subyacente. La resistividad obtenida en este estrato es mayor de $100 \Omega \mathrm{m}$, en correlación con lo obtenido por F. Morrison (Josefina, $122 \Omega$ m; Devoto, 600 $\Omega \mathrm{m})$. Los resultados obtenidos por la geofísica son coherentes con los obtenidos por Pezzi \& Mozetic (1989) en su análisis del espesor sedimentario regional Tacuarembo + Serra Geral. Los sitios de medición MT se encuentran entre las isolíneas de 1250 m y $1500 \mathrm{~m}$.

Estos resultados tienen importancia debido a que se trata de los primeros resultados en esos sitios con información por debajo de los basaltos de la de Formación Serra Geral, que suman al conocimiento del subsuelo de la Cuenca Chacoparanense.

Agradecimientos: Se agradece en forma especial al Intendente de C. Pellegrini de la Prov. De Santa Fe, Sr. Horacio Adagio y a la comunidad en general por la colaboración prestada para que estas mediciones tuvieran el éxito merecido por el esfuerzo que significa al Municipio, realizar este tipo de estudios y confiar en este Grupo de Trabajo, también al Lic. Abel Pesce por su apoyo y la confianza que nos dispensa.

\section{Referências}

Bailey R.C. \& Groom R.W. 1987. Decomposition of the Magnetotelluric Impedance Tensor Which is Useful in the Presence of Channeling. In: Soc. Expl. Geophys, Annual Internat. Mtg., Soc., 57, Expanded Abstracts, Session: MT1.1.

Berdichevsky M.N. \& Dmitriev V.I. 1976. Basic Principles of Interpretation of Magnetotelluric Sounding Curves. In: A. Adam (Ed.) Geoelectric and Geothermal Studies: Akademini Kiado. Budapest.

Chebli G.A., Mozetic M.E., Rosello E.A., Buhler M. 1999. Cuencas Sedimentarias de la Llanura Chaco - pampeana. En: R. Caminos (Ed.) Geología Argentina. Instituto de Geología y Recursos Minerales, Buenos Aires, anales, v. 29, num. 20, p. 627-644.

Diaz E., Sanguinetti J., Dallacosta O. 1993. Prospección geoelectrica de acuiferos profundos. Informe para la Municipalidad de Colón Prov. de Entre Ríos. 20 p.

Dirección de Promoción Minera e Hidrogeológica. 1992. Antecedentes
Hidrotermales de la Provincia de Entre Ríos. Informe, Paraná, Gobierno de la Provincia de Entre Ríos.

SEGEMAR 2005. Evaluación del Potencial Geotérmico en la localidad de Carlos Pellegrini, provincia de Santa Fe. Departamento de Geotermia, segunda etapa del Servicio Geológico Minero Argentino. Informe inédito.

Jones A.G. 1988. Static shift of magnetotelluric data and its removal in a sedimentary basin environment. Geophysics, 53:967-978.

Menezes P.T.L. \& Travassos J.M. 2004. EM modeling of the central northern portion of Ponta Grossa Arch, Paraná Basin Brazil. Physics of the earth and Planetary Interiors. (Article in Press).

Morrison F. 1990. Magnetotellurics Test Soundings, Paraná Basin, San Francisco - Josefina y Devoto. Informe Técnico de Electromagnetic Instrument Inc. California EE UU.

Pesce A. 2002. Thermal spas (centros termales) an economical develo- 
pment alternative along both sides of the Uruguay River. Geo Heat Center Quaterly Bulletin campus drive Klamath falls OR 97601. (translated by M. Lipmann Lawrence Berkeley National Laboratory Berkeley)

Pezzi E. \& Mozetic M. 1989. Cuencas Sedimentarias de la región chacoparanense. En: G. Chebli \& L. Spalleti (Eds.) Cuencas Sedimentarias Argentinas. Universidad Nacional de Tucumán, Instituto Superior de Correlación Geológica, Serie Correlación Geológica n ${ }^{\circ}$ 6, p 65-77, Tucumán, Argentina.

Ribeiro L.F.B., Hackspacher P.C., Ribeiro M.C.S., Hadler Neto J.C., Tello S.C.A., Iunes P.J., Franco A.O.B., Godoy D.F. 2004. Thermotectonic and fault dynamic analysis of Precambrian basement and tectonic constraints with the Parana basin. Radiation Measurements (Article in Press).

Rodi W.L \& Minster J.B. 1983. ESP/MT: An Interactive System for Two-dimensional Magnetotelluric Interpretation. Geophysics, 48:805-806.

Silva Busso A. 1999. Contribución al Conocimiento Geológico e Hidrogeológico del Sistema Acuífero Termal de la Cuenca Chacoparanense Oriental Argentina. Tesis Doctoral, Facultad de Ciencias Exactas y Naturales, (UBA).

Schenato F., Formoso M.L.L., Dudoignon P., Meunier A., Proust D., Mas A. 2003. Alteration Processes of the thick basaltic lava flow of the Parana Basin (Brazil): petrographic and mineralogical studies. Journal of South American Earth Sciences 16:423-444.

Smith J. T. 1988. Rapid inversion of multi-dimensional magnetotelluric data. University of Washington Geophysics Program Disertation, DOE/ER/13472-3.

Smith J. T. \& Booker J. R. 1991. Rapid inversion of two and three-dimensional magnetotelluric data. J. Geophys. Res., 96:3905-3922.

Thomaz Filho A., Pimentel Mizusaki A. M., Milani E. J., De Cesero P. 2000. Rifting and magmatism associated with the south America and Africa break up. Revista Brasileira de Geociencias, 30:17-19.

Vozoff K. 1991. The magnetotelluric method. In: M.N. Navighian (Ed.) Electromagnetic Methods in Applied Geophysics. Tulsa, Soc. Explor. Geophys., v. 2B. pp.: 641-711.

Manuscrito A1642 Aprovado em 18 de setembro de 2006 